\title{
Basic science: (MAY 2007)
}

1. Adamovic T, Roshani L, Chen L, Schaffer BS, Helou K, Levan G, Olsson B, Shull JD. Nonrandom pattern of chromosome aberrations in $17 \beta$-estradiol-induced rat mammary tumors: indications of distinct pathways for tumor development. Genes Chromosomes Cancer 2007; 46: 459-469.

2. Adams BD, Furneaux $H$, White BA. The microribonucleic acid (miRNA) miR-206 targets the human estrogen receptor- $\alpha \quad(E R \alpha)$ and represses $\mathrm{ER} \alpha$ messenger RNA and protein expression in breast cancer cell lines. Mol Endocrinol 2007; 21: 1132-1147.

3. Aupperlee MD, Haslam SZ. Differential hormonal regulation and function of progesterone receptor isoforms in normal adult mouse mammary gland. Endocrinology 2007; 148: 2290-2300.

4. Borgquist S, Anagnostaki L, Jirstrom K, Landberg G, Manjer J. Breast tumours following combined hormone replacement therapy express favourable prognostic factors. Int $\mathrm{J}$ Cancer 2007; 120: 2202-2207.

5. Holst F, Stahl PR, Ruiz C, Hellwinkel O, Jehan Z, Wendland $M$, Lebeau A, Terracciano L, AlKuraya K, Janicke F, Sauter G, Simon R. Estrogen receptor $\alpha$ (ESR1) gene amplification is frequent in breast cancer. Nat Genet 2007; 39: 655-660.

6. Hunter DJ, Kraft P, Jacobs KB, Cox DG, Yeager M, Hankinson SE, Wacholder S, Wang Z, Welch R, Hutchinson A, Wang J, Yu K, Chatterjee N, Orr N, Willett WC, Colditz GA, Ziegler RG, Berg CD, Buys SS, McCarty CA, Feigelson HS, Calle EE, Thun MJ, Hayes RB, Tucker M, Gerhard DS, Fraumeni JF, Hoover RN, Thomas G, Chanock SJ. A genome-wide association study identifies alleles in FGFR2 associated with risk of sporadic postmenopausal breast cancer. Nat Genet 2007; 39: 870-874.

BCO/660/2007/JW
7. Kim H, Chen JJ, Yu XH. Ubiquitin-binding protein RAP80 mediates BRCA1-dependent DNA damage response. Science 2007; 316: 1202-1205.

8. Li W, Xiao C, Vonderhaar BK, Deng CX. A role of estrogen/ER $\alpha$ signaling in BRCA1-associated tissue-specific tumor formation. Oncogene 2007 May 14; [Epub ahead of print], doi:10.1038/sj.onc.1210527.

9. Liu BL, Ordonez-Ercan D, Fan ZY, Edgerton $S M$, Yang $X H$, Thor AD. Downregulation of erbB3 abrogates erbB2-mediated tamoxifen resistance in breast cancer cells. Int $J$ Cancer 2007; 120: 1874-1882.

10. Maiello MR, D'Alessio A, De Luca A, Carotenuto A, Rachiglio AM, Napolitano M, Cito L, Guzzo A, Normanno N. AZD3409 inhibits the growth of breast cancer cells with intrinsic resistance to the EGFR tyrosine kinase inhibitor gefitinib. Breast Cancer Res Treat 2007; 102: 275-282.

11. McBryan J, Howlin J, Kenny PA, Shioda T, Martin F. ER $\alpha$-CITED1 co-regulated genes expressed during pubertal mammary gland development: implications for breast cancer prognosis. Oncogene 2007 May 7; [Epub ahead of print], doi:10.1038/sj.onc.1210468.

12. Monaco SE, Angelastro JM, Szabolcs $M$, Greene LA. The transcription factor ATF5 is widely expressed in carcinomas, and interference with its function selectively kills neoplastic, but not nontransformed, breast cell lines. Int J Cancer 2007; 120: 1883-1890.

13. Paterson AL, Pole JCM, Blood KA, Garcia MJ, Cooke SL, Teschendorff AE, Wang YZ, Chin SF, Ylstra B, Caldas C, Edwards PAW. Coamplification of $8 p 12$ and $11 q 13$ in breast cancers is not the result of a single genomic event. Genes Chromosomes Cancer 2007; 46: 427-439.

14. Pellikainen JM, Kosma VM. Activator protein-2 in carcinogenesis with a special reference to breast cancer - A mini review. Int $J$ Cancer 2007; 120: 2061-2067. 
15. Ross JS, Symmans WF, Pusztai L, Fiortobagyi GN. Standardizing slide-based assays in breast cancer: hormone receptors, HER2, and sentinel lymph nodes. Clin Cancer Res 2007; 13: 2831-2835.

16. Sabnis G, Goloubeva O, Jelovac D, Schayowitz A, Brodie A. Inhibition of the phosphatidylinositol 3-kinase/Akt pathway improves response of longterm estrogen-deprived breast cancer xenografts to antiestrogens. Clin Cancer Res 2007; 13: 2751-2757.

17. Sirvent A, Boureux A, Simon V, Leroy C, Roche $\mathrm{S}$. The tyrosine kinase Abl is required for Srctransforming activity in mouse fibroblasts and human breast cancer cells. Oncogene 2007 May 28; [Epub ahead of print], doi:10.1038/ sj.onc.1210543.

18. Sobhian B, Shao GZ, Lilli DR, Culhane AC, Moreau LA, Xia B, Livingston DM, Greenberg RA. RAP80 targets BRCA1 to specific ubiquitin structures at DNA damage sites. Science 2007; 316: 1198-1202.

19. Stacey SN, Manolescu A, Sulem P, Rafnar T, Gudmundsson J, Gudjonsson SA, Masson G, Jakobsdottir M, Thorlacius S, Helgason A, Aben KK, Strobbe LJ, Albers-Akkers MT, Swinkels DW, Henderson BE, Kolonel LN, Le Marchand L, Millastre E, Andres R, Godino J, Garcia-Prats
MD, Polo E, Tres A, Mouy M, Saemundsdottir J, Backman VM, Gudmundsson L, Kristjansson K, Bergthorsson JT, Kostic J, Frigge ML, Geller F, Gudbjartsson D, Sigurdsson H, Jonsdottir T, Hrafnkelsson J, Johannsson J, Sveinsson T, Myrdal G, Grimsson HN, Jonsson T, von Holst $S$, Werelius B, Margolin S, Lindblom A, Mayordomo JI, Haiman CA, Kiemeney LA, Johannsson OT, Gulcher JR, Thorsteinsdottir U, Kong A, Stefansson K. Common variants on chromosomes $2 q 35$ and $16 q 12$ confer susceptibility to estrogen receptor-positive breast cancer. Nat Genet 2007; 39: 865-869.

20. Ursini-Siegel J, Schade B, Muller WJ, Cardiff RD. Timeline - insights from transgenic mouse models of ERBB2-induced breast cancer. Nat Rev Cancer 2007; 7: 389-397.

21. Wang X, Liu L, Montagna C, Ried T, Deng CX. Haploinsufficiency of Parp1 accelerates Brca1associated centrosome amplification, telomere shortening, genetic instability, apoptosis, and embryonic lethality. Cell Death Differ 2007; 14: 924-931.

Prepared by

$R$ Sutherland

Cancer Research Program

Garvan Institute of Medical Research Darlinghurst, NSW, Australia 